\title{
EVAPOTRANSPIRAÇÃO DE REFERÊNCIA COM BASE NA RADIAÇÃO SOLAR GLOBAL ESTIMADA PELO MÉTODO DE BRISTOW-CAMPBELL
}

\author{
MARCO A. F. CONCEIÇÃ̃O
}

RESUMO: A quantidade de água a ser aplicada em sistemas de irrigação é normalmente calculada com base na evapotranspiração de referência (ETo) da região. Vários métodos podem ser empregados para o cálculo de ETo, sendo o de Hargreaves um dos mais adotados. Esse método utiliza valores estimados da radiação solar global (Rs) com base na diferença entre as temperaturas máxima (Tmáx) e mínima (Tmín) do ar. Entretanto, a Rs pode ser calculada por outros métodos, como o de Bristow-Campbell, que também se baseia na diferença entre Tmáx e Tmín. No presente trabalho, foram estimados valores diários da ETo pelo método de Hargreaves, em Jales - SP, com e sem o uso da equação de Bristow-Campbell para cálculo de Rs. Esses valores foram comparados aos obtidos pelo método de Penman-Monteith, que é considerado padrão para a estimativa de ETo. As avaliações consideraram os períodos úmido e seco na região. $\mathrm{O}$ uso da equação de Bristow-Campbell melhorou o desempenho do método de Hargreaves, em relação ao de Penman-Monteith, sendo que durante o período seco do ano o desempenho foi superior ao do período úmido.

PALAVRAS-CHAVE: Hargreaves, Penman-Monteith, irrigação.

\section{REFERENCE EVAPOTRANSPIRATION BASED ON SOLAR RADIATION ESTIMATED BY THE BRISTOW-CAMPBELL MODEL}

\begin{abstract}
The amount of water used in irrigation systems is usually calculated using the local reference evapotranspiration (ETo) values. Several methods can be used for ETo calculation, and Hargreaves method is one of the most popular. This method uses estimated values of global solar radiation (Rs), based on the difference between the maximum (Tmax) and minimum (Tmin) air temperature. However, the Rs may be calculated by other methods, such as the Bristow-Campbell, which also is based on the difference between Tmax and Tmin. In this study were estimated daily values of ETo by the method of Hargreaves in Jales - SP, Brazil, with and without the use of the Bristow-Campbell equation to calculate the Rs. These values were compared with those from the Penman-Monteith method, which is considered standard for the estimation of ETo. The evaluations had considered the wet and dry periods in the region. The use of the Bristow-Campbell equation improved the performance of the Hargreaves method, in relation to the Penman-Monteith, and the performance during the dry period of the year was higher than that of the wet season.
\end{abstract}

KEYWORDS: Hargreaves, Penman-Monteith, irrigation.

\section{INTRODUÇÃO}

A evapotranspiração de referência (ETo) é um parâmetro fundamental para a estimativa das necessidades hídricas das culturas, sendo desejável que se tenha um método que estime a ETo com boa precisão, a partir de dados meteorológicos fáceis de serem obtidos (VILLA NOVA \& PEREIRA, 2006). Embora o método de Penman-Monteith (EToPM) seja considerado, atualmente, como padrão para a estimativa de ETo (ORTEGA-FARIAS et al., 2009), o seu uso é limitado, uma vez que são necessárias variáveis meteorológicas nem sempre disponíveis. Por essa razão, outros métodos que empregam um menor número de variáveis são, muitas vezes,

\footnotetext{
${ }^{1}$ Pesquisador A, Embrapa Uva e Vinho, Estação Experimental de Viticultura Tropical, Caixa Postal 241, Jales - SP, Fone: (0XX17) 3632-9666, marcoafc@ cnpuv.embrapa.br. 
utilizados na estimativa de ETo. Entre esses, destaca-se o de Hargreaves (HARGREAVES \& ALLEN, 2003).

Nesse método, a ETo é calculada com base em valores da temperatura média do ar e da radiação solar global (Rs), que é estimada a partir da diferença entre as temperaturas máxima (Tmáx) e mínima (Tmín) do ar. Essa estimativa considera que, na presença de nuvens, quando os valores de Rs são menores, essa diferença (Tmáx-Tmín) também tende a ser menor (ALLEN et al., 1998).

Diferentes autores propuseram modificações e ajustes na equação de Hargreaves (eq.3), buscando melhor adequação às condições de cada local. Alguns trabalhos visaram á calibração dos coeficientes empíricos da equação (BORGES \& MENDIONDO, 2007; FOOLADMAND \& HAGHIGHAT, 2007; TRAJKOVIC, 2007; BAUTISTA et al., 2009; VEGA \& JARA, 2009), enquanto outros inseriram elementos meteorológicos no modelo, como a precipitação pluvial (DROOGERS \& ALLEN, 2002) ou o vento (GAVILÁN et al., 2006). Nenhum desses trabalhos, entretanto, modificou a base da equação de Hargreaves, que é o método de estimativa de Rs a partir da diferença entre Tmáx e Tmín.

Outras equações, entretanto, também podem ser utilizadas para estimar a Rs a partir dessa diferença, como a de Bristow-Campbell (BRISTOW \& CAMPBELL, 1984). CONCEIÇÃO \& MARIN (2007) verificaram que esse método (RsB) proporcionou melhores estimativas da radiação solar global do que o de Hargreaves ( $\mathrm{RsH}$ ), em diferentes regiões do Brasil. O emprego dos valores de RsB no lugar de RsH na equação de Hargreaves poderia, assim, melhorar a estimativa de ETo.

Para testar essa hipótese, buscou-se, no presente trabalho, comparar os valores estimados de ETo pelo método-padrão de Penman-Monteith (EToPM) com os obtidos pelo método original de Hargreaves (empregando-se $\mathrm{RsH}$ ) e pelo método de Hargreaves modificado (empregando-se RsB).

\section{MATERIAL E MÉTODOS}

Para calcular a ETo pelo método de Penman-Monteith, foi utilizada a seguinte expressão:

$$
\begin{aligned}
& \mathrm{EToPM}=0,408 \mathrm{~s}(\mathrm{Rn}-\mathrm{G})+\gamma 900 \mathrm{U}_{2}\left(\mathrm{e}_{\mathrm{s}}-\mathrm{e}_{\mathrm{a}}\right) \\
& \text { (Tmed + 273) } \\
& \mathrm{s}+\gamma\left(1+0,34 \mathrm{U}_{2}\right)
\end{aligned}
$$

em que,

EToPM - evapotranspiração de referência pelo método de Penman-Monteith, $\mathrm{mm} \mathrm{dia}^{-1}$;

$\mathrm{S}$ - declividade da curva de pressão de vapor em relação à temperatura, $\mathrm{kPa}^{\circ} \mathrm{C}^{-1}$;

$\mathrm{Rn}$ - radiação líquida total diária, $\mathrm{MJ} \mathrm{m}^{-2} \mathrm{dia}^{-1}$;

$\mathrm{G}$ - fluxo total diário de calor no solo, $\mathrm{MJ} \mathrm{m}^{-2} \mathrm{dia}^{-1}$;

$\gamma$ - coeficiente psicrométrico, $\mathrm{kPa}^{\circ} \mathrm{C}^{-1}$;

$\mathrm{U}_{2}$ - velocidade do vento a $2 \mathrm{~m}$ de altura, $\mathrm{m} \mathrm{s}^{-1}$;

$\mathrm{e}_{\mathrm{s}}$ - pressão de saturação de vapor, $\mathrm{kPa}$;

$\mathrm{e}_{\mathrm{a}}$ - pressão parcial de vapor, $\mathrm{kPa}, \mathrm{e}$

Tméd - temperatura média do ar, ${ }^{\circ} \mathrm{C}$.

O fluxo de calor no solo $(\mathrm{G})$ foi considerado igual a zero, valor que pode ser assumido para períodos diários (ALLEN et al., 1998; BACK, 2007). A radiação líquida (Rn) foi estimada empregando-se a seguinte expressão, ajustada para as condições regionais (CONCEIÇÃO, 2006): 


$$
\mathrm{Rn}=0,653 \mathrm{Rs}
$$

em que,

$$
\text { Rs - radiação solar global, } \mathrm{MJ} \mathrm{m}^{-2} \mathrm{dia}^{-1} \text {. }
$$

A eq.(2) $\left(n=710 ; R^{2}=0,90\right)$ foi empregada para todos os dias do ano.

O método de Hargreaves para a estimativa de ETo pode ser descrito da seguinte forma (ALLEN et al., 1998; HARGREAVES \& ALLEN, 2003):

$$
\text { EToH }=0,0009 \text { Ra }(\text { Tmáx }- \text { Tmín })^{0,5}(\text { Tméd + 17,8) }
$$

em que,

EToH - evapotranspiração de referência pelo método de Hargreaves, $\mathrm{mm} \mathrm{dia}^{-1}$;

$\mathrm{Ra}$ - radiação solar incidente no topo da atmosfera, $\mathrm{MJ} \mathrm{m}^{-2} \mathrm{dia}^{-1}$;

Tmáx - temperatura máxima do ar, ${ }^{\circ} \mathrm{C}$, e

Tmín - temperatura mínima do ar, ${ }^{\circ} \mathrm{C}$.

O coeficiente empírico 0,0009 é empregado para valores de $\mathrm{Ra}$ em $\mathrm{MJ} \mathrm{m}^{-2} \mathrm{dia}^{-1}$. No original, em que os valores de Ra estão em mm dia ${ }^{-1}$, o coeficiente é igual a 0,0023.

$\mathrm{Na}$ eq.(3) está inserida a estimativa da radiação solar global que, para regiões continentais, é calculada empregando-se a seguinte expressão (HARGREAVES \& ALLEN, 2003):

$$
\mathrm{RsH}=0,16 \text { (Tmáx - Tmín })^{0,5} \mathrm{Ra}
$$

em que,

$\mathrm{RsH}$ - radiação solar global estimada pelo método de Hargreaves, $\mathrm{MJ} \mathrm{m}^{-2} \mathrm{dia}^{-1}$.

Assim, a eq.(3) pode ser reescrita da seguinte forma:

$$
\mathrm{EToH}=0,0056 \mathrm{RsH}(\text { Tméd }+17,8)
$$

Já o modelo de Bristow-Campbell para a estimativa de Rs pode ser descrito da seguinte maneira (CONCEIÇÃO \& MARIN, 2007):

$$
\text { RsB = 0,7 [1 - exp(-0,005 (Tmáx - Tmín) } \left.\left.{ }^{2,4}\right)\right] \text { Ra }
$$

em que,

RsB - radiação solar global estimada pelo modelo de Bristow-Cambell, $\mathrm{MJ} \mathrm{m}^{-2} \mathrm{dia}^{-1}$.

Substituindo-se RsB na eq.(5), no lugar de RsH, obtém-se a expressão:

$$
\mathrm{EToB}=0,0056 \mathrm{RsB}(\text { Tmed }+17,8)
$$

Em que,

EToB - evapotranspiração de referência utilizando-se de RsB, mm dia ${ }^{-1}$.

Foram utilizados registros meteorológicos diários coletados na estação automática da Embrapa Uva e Vinho, em Jales-SP $\left(20^{\circ} 10^{\prime} \mathrm{S}, 50^{\circ} 35^{\prime} \mathrm{W}, 455 \mathrm{~m}\right)$, referentes aos anos de 2004 a 2007. Para a coleta das variáveis meteorológicas, foi empregado um sistema automático de aquisição de dados modelo CR-510, da Campbell ${ }^{\odot}$, com registros efetuados a cada 15 minutos e totalizados diariamente. Foram utilizados piranômetros modelo Licor LI-200X, para a determinação de Rs, e sensores de temperatura e umidade relativa modelo Rotronic Hygroclip 525. A velocidade do vento foi medida usando um anemômetro da marca Young, instalado a $2,0 \mathrm{~m}$ de altura. 
As avaliações consideraram todos os dados diários entre 2004 e 2007 e, também, separadamente os valores relativos aos períodos úmido (de novembro a março) e seco (de abril a setembro) na região.

As comparações entre os valores estimados com os de EToPM foram realizadas empregando-se regressões lineares, obtendo-se os coeficientes de determinação $\left(\mathrm{R}^{2}\right)$. Também foi utilizado o coeficiente de desempenho (c) proposto por CAMARGO \& SENTELHAS (1997), que corresponde à multiplicação do coeficiente de correlação (r) pelo coeficiente de exatidão (d), que pode ser escrito da seguinte forma:

$$
\mathrm{d}=1-\left\{\sum(\mathrm{Xi}-\mathrm{Yi})^{2} / \sum(|\mathrm{Xi}-\mathrm{Ym}|+|\mathrm{Yi}-\mathrm{Ym}|)^{2}\right\}
$$

em que,

d - coeficiente de exatidão, adimensional;

$\mathrm{Xi}$ - valores de EToH e EToB, mm dia ${ }^{-1}$;

Yi - valores de EToPM, mm dia ${ }^{-1}$, e

Ym - média dos valores de EToPM, mm dia ${ }^{-1}$.

O desempenho foi classificado como ótimo para valores de c maiores que 0,85 ; como muito bom para valores entre 0,76 e 0,85 ; como bom para valores entre 0,66 e 0,75 ; como regular para valores entre 0,51 e 0,65 ; como ruim para valores entre 0,41 e 0,50 ; e como péssimo para valores inferiores a 0,40 .

\section{RESULTADOS E DISCUSSÃO}

Os valores médios de ETo e as relações percentuais dos diferentes métodos em relação a EToPM estão apresentados na Tabela 1. Observa-se que os valores médios estimados superestimaram os valores de EToPM em todas as condições. As maiores diferenças ocorreram no período úmido (novembro a março), quando as médias de EToH e EToB se apresentaram entre $17,18 \%$ e $17,51 \%$ superiores a EToPM, respectivamente. Já no período seco (abril a outubro), as diferenças em relação à EToPM foram menores, com superestimativas médias entre $1,22 \%$ e $4,78 \%$ para EToH e EToB, respectivamente.

TABELA 1. Valores médios (Média) da evapotranspiração de referência estimada empregandose as equações de Hargreaves (EToH), de Bristow-Campbell (EToB) e de PenmanMonteith (EToPM), considerando-se todos os dados (Geral) e os períodos úmido e seco. Jales - SP, 2004-2007. Average values (Média) of reference evapotranspiration estimated using Hargreaves (EToH), Bristow-Campbell (EToB) and Penman-Monteith (EToPM) equations, considering all data (Geral) and the wet (Úmido) and dry (Seco) periods. Jales - SP, Brazil, 20042007.

\begin{tabular}{lcrc}
\hline Índice & EToH & EToB & EToPM \\
\hline Média geral $\left(\mathrm{mm} \mathrm{dia}^{-1}\right)$ & 4,51 & 4,59 & 4,16 \\
ETo/EToPM geral $(\%)$ & 8,29 & 10,16 & - \\
\hline Média úmido $\left(\mathrm{mm} \mathrm{dia}^{-1}\right)$ & 5,15 & 5,14 & 4,38 \\
ETo/EToPM Úmido $(\%)$ & 17,51 & 17,18 & - \\
\hline Média seco $\left(\mathrm{mm} \mathrm{dia}^{-1}\right)$ & 4,06 & 4,20 & 4,01 \\
ETo/EToPM Seco $(\%)$ & 1,22 & 4,78 & - \\
\hline
\end{tabular}

Os coeficientes de determinação $\left(\mathrm{R}^{2}\right)$ empregando-se EToB foram superiores aos obtidos empregando-se EToH, tanto para a série total de dados (Figura 1), quanto para os períodos úmido (Figura 2) e seco (Figura 3). Para EToH, o maior valor de $\mathrm{R}^{2}$ foi obtido no período úmido, enquanto para EToB não houve diferença expressiva entre os diferentes períodos. Observa-se 
que a EToB permitiu estimar com maior exatidão os valores inferiores a $2,0 \mathrm{~mm} \mathrm{dia}^{-1}$, do que EToH (Figuras 1 a 3).
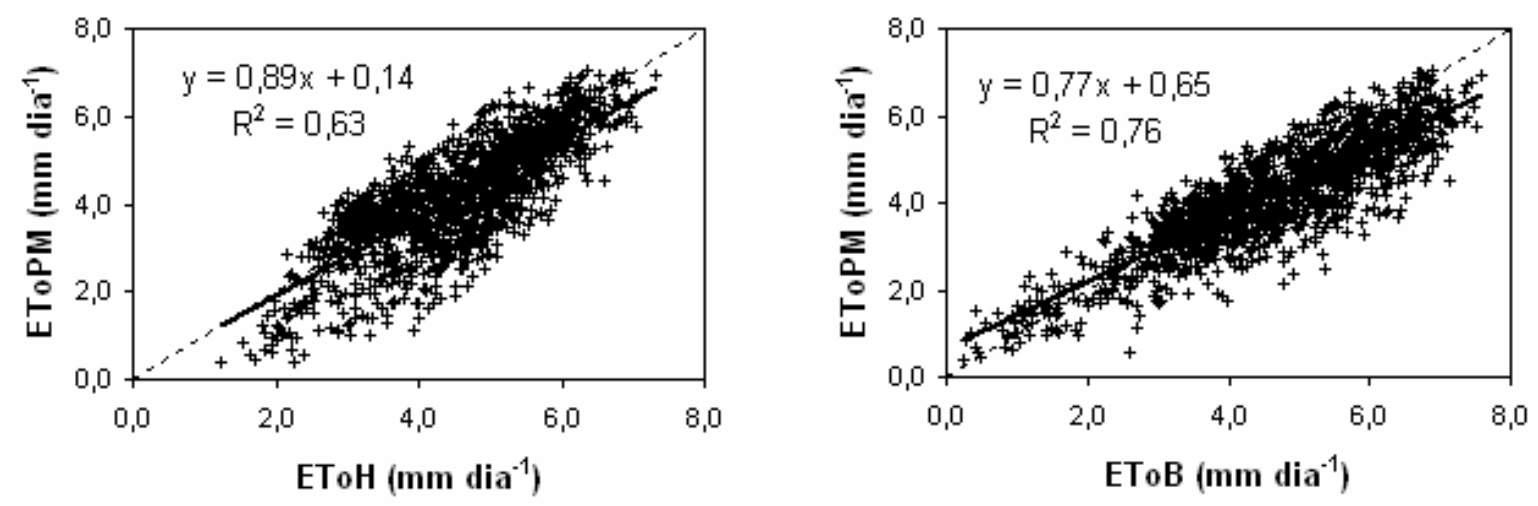

FIGURA 1. Relações entre valores da evapotranspiração de referência estimada pelo método de Penman-Monteith (EToPM) e empregando-se as equações de Hargreaves (EToH) e de Bristow-Campbell (EToB), para o período anual. Jales-SP, 2004-2007. Relationship between reference evapotranspiration values estimated by the Penman-Monteith (EToPM) method and using the Hargreaves (EToH) and Bristow-Campbell (EToB) equations, for the annual period. Jales - SP, 20042007.
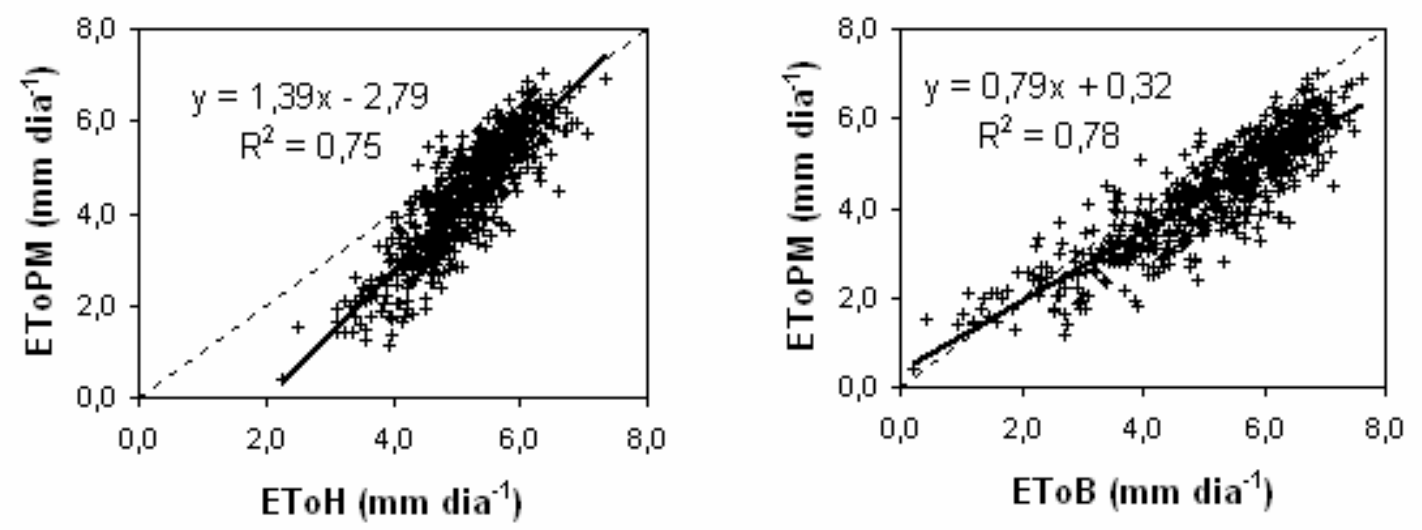

FIGURA 2. Relações entre valores da evapotranspiração de referência estimada pelo método de Penman-Monteith (EToPM) e empregando-se as equações de Hargreaves (EToH) e de Bristow-Campbell (EToB) durante o período úmido, de novembro a março. Jales-SP, 2004-2007. Relationship between reference evapotranspiration values estimated by the Penman-Monteith (EToPM) method and using the Hargreaves (EToH) and Bristow-Campbell (EToB) equations, for the wet period, from November to March. Jales - SP, 2004-2007. 

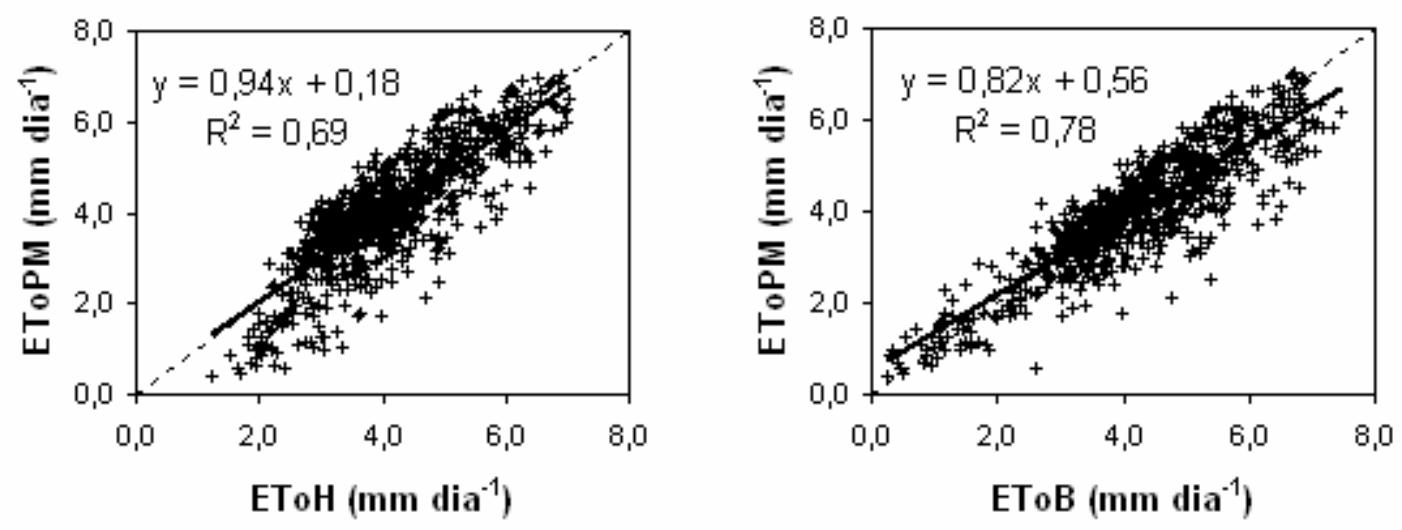

FIGURA 3. Relações entre valores da evapotranspiração de referência estimada pelo método de Penman-Monteith (EToPM) e empregando-se as equações de Hargreaves (EToH) e de Bristow-Campbell (EToB) durante o período seco, de abril a outubro. Jales-SP, 2004-2007. Relationship between reference evapotranspiration values estimated by the Penman-Monteith (EToPM) method and using the Hargreaves (EToH) and Bristow-Campbell (EToB) equations, for the dry period, from April to October. Jales - SP, 2004-2007.

Os coeficientes de correlação, exatidão e desempenho dos modelos em relação a EToPM estão apresentados na Tabela 2. As maiores diferenças entre os períodos úmido e seco ocorreram em relação aos coeficientes de exatidão (d).

TABELA 2. Coeficientes de correlação (r), de exatidão (d) e de desempenho (c) da evapotranspiração de referência (ETo) estimada empregando-se as equações de Hargreaves $($ EToH) e de Bristow-Campbell (EToB) em relação à ETo estimada pelo método de Penman-Monteith (EToPM), considerando-se todos os dados (Geral) e os períodos úmido e seco. Jales - SP. 2004-2007. Correlation (r), accuracy (d) and performance (c) coefficients for reference evapotranspiration (ETo) estimated using the Hargreaves (EToH) and Bristow-Campbell (EToB) equation in relation to the estimated PenmanMonteith values (EToPM), considering all data (Geral) and the wet (Úmido) and dry (Seco) periods. Jales - SP. 2004-2007.

\begin{tabular}{|c|c|c|c|c|c|c|}
\hline \multirow{2}{*}{ Coeficiente } & \multicolumn{2}{|c|}{ Geral } & \multicolumn{2}{|c|}{ Úmido } & \multicolumn{2}{|c|}{ Seco } \\
\hline & EToH & EToB & EToH & EToB & EToH & EToB \\
\hline$r$ & 0,79 & 0,87 & 0,87 & 0,88 & 0,83 & 0,88 \\
\hline d & 0,87 & 0,91 & 0,78 & 0,86 & 0,91 & 0,93 \\
\hline $\mathrm{c}$ & 0,69 & 0,79 & 0,67 & 0,76 & 0,76 & 0,82 \\
\hline Classificação & bom & muito bom & bom & muito bom & muito bom & muito bom \\
\hline
\end{tabular}

No período úmido, os desempenhos dos modelos foram inferiores aos do período seco. Isso poderia ser esperado, uma vez que os modelos avaliados se baseiam na eq.(3), que foi desenvolvida empiricamente para as condições semiáridas da Califórnia (HARGREAVES \& ALLEN, 2003). Além disso, normalmente, no período chuvoso, as diferenças entre Tmáx e Tmín são menores do que no período seco, e as estimativas de Rs apresentam maior eficácia quando essas diferenças são maiores (LIU et al., 2009).

$\mathrm{O}$ uso de EToH proporcionou um desempenho classificado como bom, considerando-se os dados em geral e o período úmido; e como muito bom no período seco. Já os valores de EToB apresentaram desempenhos classificados como muito bons em todos os períodos (Tabela 2). Esse resultado está relacionado ao melhor desempenho do modelo de Bristow-Campbell (eq.(6)), em 
relação ao de Hargreaves (eq.(4)), na estimativa de Rs na região em que foram feitas as avaliações (CONCEIÇÃO \& MARIN, 2007).

Observa-se que, quanto mais próximos os valores estimados de Rs estiverem em relação aos valores medidos, melhor será o desempenho do modelo. De fato, a substituição de RsH (estimado) por Rs (medido) na eq.(5) acarreta uma elevação considerável no seu desempenho em relação ao método de Penman-Monteith, sendo que, no período seco, o valor de $\mathrm{R}^{2}$ chega a 0,92 (Figura 4), enquanto o coeficiente de desempenho (c) alcança 0,96, classificando-o como ótimo. Teoricamente, esses seriam os valores máximos que a eq.5 poderia alcançar para esse período do ano.

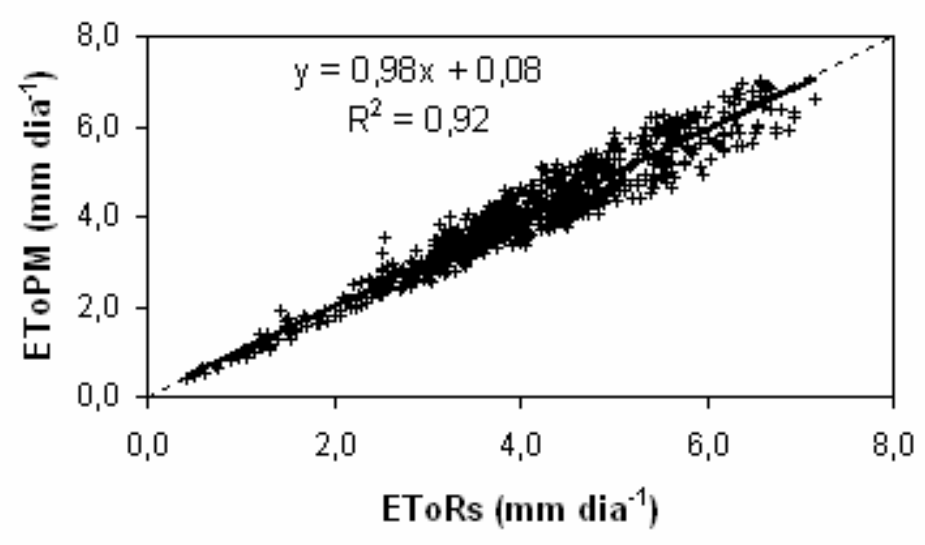

FIGURA 4. Relações entre a evapotranspiração diária estimada pelo método de Hargreaves, empregando-se valores medidos da radiação solar global (EToRs) e a evapotranspiração diária estimada pelo método de Penman-Monteith (EToPM) durante o período seco, de abril a outubro. Jales-SP, 2004-2007. Relationship between daily evapotranspiration estimated by the Hargreaves method, using measured values of global solar radiation (EToRs) and daily evapotranspiration estimated by Penman-Monteith (EToPM) during the dry season, from April to October. Jales-SP, 2004-2007.

O uso de EToB (eq.(7)) pode, assim, ser indicado para a região noroeste paulista, principalmente durante o período seco do ano, época em que a irrigação é mais utilizada. O seu emprego é bastante simples, sendo apropriado aos produtores que dispõem apenas de informações diárias da temperatura máxima e mínima do ar.

\section{CONCLUSÕES}

A estimativa da evapotranspiração de referência empregando-se o modelo de Bristow-Campbell para estimar a radiação solar global proporcionou desempenho superior ao obtido com o método original de Hargreaves.

O desempenho dos modelos durante o período seco do ano (abril a outubro) foi superior ao do período úmido (novembro a março).

\section{REFERÊNCIAS}

ALLEN R.G.; PEREIRA, L.; RAES, D.; SMITH, M. Crop evapotranspiration: guidelines for computing crop water requirements. Rome: FAO, 1998. (Irrigation and Drainage Paper, 56).

BACK, A.J. Variação da evapotranspiração de referência calculada em diferentes intervalos de tempo. Engenharia Agrícola, Jaboticabal, v.27, n.1, p.139-145, 2007.

BAUTISTA, F.; BAUTISTA, D.; DELGADO-CARRANZA, C. Calibration of the equations of Hargreaves and Thornthwaite to estimate the potential evapotranspiration in semi-arid and 
subhumid tropical climates for regional applications. Atmósfera, México, v.22, n.4, p.331-348, 2009.

BORGES, A.C.; MENDIONDO, E.M. Comparação entre equações empíricas para estimativa da evapotranspiração de referência na Bacia do Rio Jacupiranga. Revista Brasileira de Engenharia Agrícola e Ambiental, Campina Grande, v.11, n.3, p.293-300, 2007.

BRISTOW, K.L.; CAMPBELL, G.S. On the relationship between incoming solar radiation and daily maximum and minimum temperature. Agricultural and Forest Meteorology, Amsterdam, v.31, p.159-166, 1984.

CAMARGO, A P. de; SENTELHAS, P.C. Avaliação do desempenho de diferentes métodos de estimativa da evapotranspiração potencial no Estado de São Paulo, Brasil. Revista Brasileira de Agrometeorologia, Santa Maria, v.5, n.1, p.89-97, 1997.

CONCEIÇÃO, M.A.F. Modelos de estimativa do saldo de radiação na região noroeste do Estado de São Paulo. In: CONGRESSO NACIONAL DE IRRIGAÇÃO E DRENAGEM, 16., 2006, Goiânia. Anais... Viçosa - MG: Contexto/CENTEV/UFV, 2006. 6 p. CD-ROM.

CONCEIÇÃO, M.A.F.; MARIN, F.R. Avaliação de modelos para a estimativa de valores diários da radiação solar global com base na temperatura do ar. Revista Brasileira de Agrometeorologia, Piracicaba, v.15, n.1, p.103-108, 2007.

DROOGERS, P.; ALLEN, R.G. Estimating reference evapotranspiration under inaccurate data conditions. Irrigation and Drainage Systems, Amsterdam, v.16, p.33-45, 2002.

FOOLADMAND, H.R.; HAGHIGHAT, M. Spatial and temporal calibration of Hargreaves equation for calculating monthly ETo based on Penman-Monteith method. Irrigation and Drainage, Hoboken, v.56, p.439-449, 2007.

GAVILÁN, P.; LORITE, I.J.;TORNERO, S.; BERENGENA, J. Regional calibration of Hargreaves equation for estimating reference ET in a semiarid environment. Agricultural Water Management, Amsterdam, v.81, p.257-281, 2006.

HARGREAVES, G.H.; ALLEN, R.G. History and evaluation of Hargreaves evapotranspiration equation. Journal of Irrigation and Drainage Engineering, Reston, v.129, n.1, p.53-63, 2003.

LIU X.; MEI, X.; LI, Y.; WANGA, Q.; JENSEN, J.R.; ZHANG, Y.; PORTER, J.R. Evaluation of temperature-based global solar radiation models in China. Agricultural and Forest Meteorolgy, Amsterdam, v.149, p.1.433-1.446, 2009.

ORTEGA-FARIAS, S.; IRMAK, S.; CUENCA, R.H. Special issue on evapotranspiration measurement and modeling. Irrigation Science, New York, v.28, p.1-3, 2009.

TRAJKOVIC, S. Hargreaves versus Penman-Monteith under humid conditions. Journal of Irrigation and Drainage Engineering, Reston, v.133, n.1, p.38-42, 2007.

VEGA, E.C.; JARA, J.C. Estimación de la evapotranspiración de referencia para dos zonas (costa y región andina) del Ecuador. Engenharia Agrícola, Jaboticabal, v.29, n.3, p.390-403, 2009.

VILLA NOVA, N.A.; PEREIRA, A.B. Ajuste do método de Priestley-Taylor às condições climáticas locais. Engenharia Agrícola, Jaboticabal, v.26, n.2, p.395-405, 2006. 